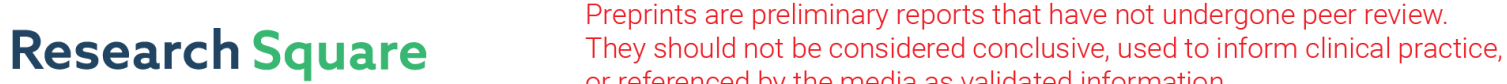 or referenced by the media as validated information. \\ Evidence of coupled oscillator to wave packet dynamics in human brain
}

\section{Mayukha Pal}

Asea Brown Boveri Company https://orcid.org/0000-0001-6037-1338

Neeraj .

Indian Institute of Science Education and Research Kolkata

Prasanta Panigrahi ( $\sim$ pprasanta@iiserkol.ac.in )

Indian Institute of Science Education and Research Kolkata https://orcid.org/0000-0003-1365-5252

\section{Research Article}

Keywords: Brain EEG signal, Epilepsy, Damped driven oscillator, Limit cycle, Wave packet, Coherence, Fourier power spectra, Unstable periodic orbits, Heisenberg Model, Neutral Turbulence.

Posted Date: December 1st, 2020

DOl: https://doi.org/10.21203/rs.3.rs-86186/v2

License: (c) (i) This work is licensed under a Creative Commons Attribution 4.0 International License. Read Full License

Version of Record: A version of this preprint was published at IEEE Access on February 15th, 2022. See the published version at https://doi.org/10.1109/ACCESS.2022.3151692. 


\title{
Evidence of coupled oscillator to wave packet dynamics in human brain
}

Mayukha Pal ${ }^{1}$, Neeraj ${ }^{2}$, Prasanta K. Panigrahi ${ }^{2, \$}$

${ }^{1}$ ABB Ability Innovation Center, Asea Brown Boveri Company, Hyderabad 500084, India.

${ }^{2}$ Indian Institute of Science Education and Research Kolkata, Mohanpur 741246, India.

\section{${ }^{\$}$ Corresponding author: \\ Prof. Prasanta K. Panigrahi \\ Professor, Department of Physical Sciences \\ Indian Institute of Science Education and Research Kolkata, \\ Mohanpur-741 246, West Bengal, India.}

Tele: $+91-9748918201$

Email:pprasanta@iiserkol.ac.in

\begin{abstract}
Clear evidence of oscillator dynamics to wave packet collapse and revival in the brain EEG signals is demonstrated for the first time, with distinct differences in healthy individuals and epileptic patients. The low frequency components exhibit single oscillator type behavior in a phase-space description, with the potentials as coordinates and their instantaneous changes as the corresponding velocities. The closed paths reveal periodic motion, well described by one linear oscillator or possibly coupled ones with limit cycle dynamics and synchronization at the macroscopic level. The epileptic patients reveal dynamical features of bi-stability, originating from non-linearity, a prominent feature in the signals from the epileptogenic zone and much enhanced during the periods of seizure. Analogy from the phase-space of oscillator dynamics reveals dominance of potential energy in the signals from the epileptogenic zone, as also in the patients during occurrence of seizure. The acceleration, arising from the change of velocity, is found to be particularly strong in epileptic patients, when the phase space shows bi-stability and bursty behavior. Wave behavior with characteristics of superposition emerges in the frequency range corresponding to the observed unstable periodic orbits, that appear at the boundary of alpha and beta waves of $8-14 \mathrm{~Hz}$, centered at $10 \mathrm{~Hz}$. A modulated carrier wave is observed for all subjects at $18 \mathrm{~Hz}$, higher in width for patients. Coherent wave dynamics, with interference playing a key role in the wave packet collapse and revival, is observed starting from $18 \mathrm{~Hz}$, with the coherence getting significantly enhanced around 40-45Hz. Mechanism of intra frequency energy transfer is shown to be neutral turbulence.
\end{abstract}

Keywords: Brain EEG signal, Epilepsy, Damped driven oscillator, Limit cycle, Wave packet, Coherence, Fourier power spectra, Unstable periodic orbits, Heisenberg Model, Neutral Turbulence. 


\section{Significance}

Observation of oscillator behavior and wave phenomena characterized by superposition, wave packet collapse and revival are unequivocally established through a phase space approach, involving potential and its instantaneous changes, traditionally employed in studying electrical circuits. Clear observation of bi-stability, bursty dynamics, unstable periodic orbits and strong intra-band energy transfer through neutral turbulence are shown. Wave packet collapse and revival in brain EEG signals, with carrier wave modulation is demonstrated for the first time, unveiling the domain of mono frequency oscillations, interference and emergent coherent wave packet dynamics. The instantaneous approach reveals finer structures in phase space, as compared to global nonlinear time series analysis through embedding dimension, often hampered by insufficient data length and boundary effects.

\section{Introduction}

Human brain is central to all cognitive and sensory stimuli that control the body functions, producing complex biopotential signals. Dynamical behavior of the brain is reflected in the recording of the electroencephalogram (EEG) signals, a much-used approach to measure the neurophysiological activity of the brain in the form of electrical signals [1-5]. Seizure is a brain disease which occurs spontaneously, due to abnormal electrical activity occurring temporarily in nerve cells. Nearly $0.6-0.8 \%$ of the world's population is reported to be suffering from epilepsy, with symptoms showing seizures [6]. Usually EEG signals contain multiple frequency bands: the frequencies of the gamma waves are greater than $30 \mathrm{~Hz}$, those of the beta waves range from 13$30 \mathrm{~Hz}$, with alpha lying in $8-12 \mathrm{~Hz}$, theta in $4-8 \mathrm{~Hz}$, and delta being less than $4 \mathrm{~Hz}$. During resting state, meditation or quiet periods of the brain, alpha waves are the dominant modes. It is reported that different brain states can be characterized by the occurrence of distinct oscillatory patterns from the EEG recordings in different frequency bands [7].

In nature, the dynamical behaviors are separated into two categories: particle and wave dynamics. Classical particle dynamics is described by Newtonian laws in terms of trajectory, well described in phase-space using coordinates and their instantaneous changes, the momenta. The simplest example of particle motion is that of a spring described by Hooke's law, resulting in mono frequency sinusoidal motion. Its generalization, the damped driven oscillator finds much application in describing diverse physical phenomena, including those of potential generating electrical circuits, the key elements in brain dynamics. Coupled oscillators can show synchronized mono frequency behavior, resembling a single oscillator as also that of limit cycle. The wave dynamics is characterized by superposition, showing interference effects. This leads to an increase in amplitude, when waves interfere constructively and its decrease, arising from destructive interference, collapse and revival of wave packets being one such physical manifestation and the modulation of a carrier wave with higher frequency waves being another. All of these dynamical phenomena can be inferred through a phase-space approach by studying the brain potential values and its changes as the corresponding velocities (momenta for unit mass). Fourier space power 
spectra can faithfully reflect both periodic dynamics and self-similar behaviors, as also the unstable periodic orbits [8-10].

The measured brain potentials have been physically modeled earlier to be originating from a single oscillator at low frequency or multiple coupled ones, possibly with non-linearity at higher frequency range, leading both to synchronization and chaotic dynamics in different physiological conditions [11-15]. Non-linear time series analysis has been employed to infer about the fractal characteristics and to identify the effective degrees of freedom through the phenomenon of phase space recurrence and the largest Kolmogorov exponent, showing the diverging trajectories underlying chaotic behavior. The fact that the basic electronic unit for potential generation, incorporating capacitive, inductive and resistive components, as well as the source, is modeled as a damped driven oscillator, indicates the use of the brain potential and its changes in time as coordinate and velocity, whose variations in time will quantify the underlying forces in a Newtonian approach. Here, we take recourse to this instantaneous approach, which clearly shows the presence of a particle type single oscillator behavior at low frequencies, with closed trajectories in phase space, indicative of either oscillator dynamics or limit cycle behavior. Bi-stability and coupled oscillations manifest at still higher frequencies, both in the epileptic zones as well as during the periods of seizure. Prior to that, one observes decrease in kinetic energy with simultaneous increase in the potential energy for both of the above cases. The wave behavior begins to emerge from $18 \mathrm{~Hz}$ and higher frequencies, showing interference and wave packet collapse and revival. The Fourier domain analysis clearly reveals these behaviors, as well as that of unstable periodic orbits in the boundary of regular and chaotic dynamics, the latter showing power law decay signifying self-similarity. The local time-frequency domain wavelet analysis reveals the nature of wave packet dynamics as carrier wave modulation and wave packet collapse and revival. The process of inter modal energy transfer is identified as Heisenberg's neutral turbulence. This process is found to be operative in a large frequency range for the normal brains and in the low frequency domain for the seizure patients.

There are a number of studies devoted to the analysis of the multiscale neuronal behavior [16-18]. Often these analyses use sophisticated nonlinear models and methods of study, whose accuracy may be curtailed due to finite data length and the intrinsic limitation of the model itself. Therefore, it is pertinent to employ reliable methods, well tested in diverse systems. It has been pointed out earlier that the brain dynamics at a global level may be simple enough to be tractable [19-25]. The instantaneous phase-space approach used here reveals this behavior quite transparently, as well as the subtle differences between the patients and healthy individuals. In the Fourier domain, one observes unstable periodic orbits (UPOs), earlier evoked for explaining observed features in brain dynamics. Significantly, the UPO is found to mark the emergence of wave dynamics, characterized by carrier wave modulation and wave packet collapse and revival. The time frequency analysis using Morlet wavelets precisely points out the local bursty behavior and the neutral turbulence being the mode of intra frequency energy transfer. The dynamics of the brain activity can evolve 
to either a fixed-point attractor, corresponding to a normal brain or to a limit cycle attractor corresponding to the pathological spike wave discharges observed in epileptic brains [26-28].

\section{Results and Discussion}

We first demonstrate the oscillator behavior in the low frequency components of the EEG signals. The phase space description reveals mono-frequency modulations, representable by one linear oscillator at the macroscopic level. The epileptic patients show dynamical features of coupled oscillators, with non-linearity manifesting in the observed bifurcation and limit cycle behavior in phase-space. In particular, the bi-stability is found to be stronger in the epileptogenic zone and during the periods of seizure. The phase space plots for the normalized first channel recordings shown in Fig 1 as a characteristic example, clearly shows the existence of single particle oscillatorlike behavior at macroscopic level with closed orbits of varying size. The phase space plots in Figs 1c, and 1d possibly reveal limit cycle and bi-stability behavior, stronger in the epileptogenic zone and during the periods of seizure. It is worth emphasizing that secondary limit cycle behavior during the seizure is the characteristic feature earlier reported for rats [25]. The multiple converging loops in the phase space shows the multi-periodic nature of the dynamics, with the random phase change of the periodic orbits indicating the intermittency behavior of the subject under study. The limit cycle behavior at each of the brain frequency bands appears to provide a more accurate representation of the EEG signal than the one based on chaotic phenomena.

From Fig 1, it is further observed that the phase space plot for the epileptogenic zone is prolonged with compact width, indicating dominance of potential energy. The release of the same may be an indication of bursty behavior as observed during the period of seizure. The energy stored here is possibly getting transferred to intra frequency bands of the brain, as observed and reported first in this work. From Fig 1g, the observed acceleration in phase space reveals the large amplitude bursty behavior for the epileptic case, with higher potential and lower kinetic energy as also the phenomena of interband energy transfer. It is worth emphasizing that our phase space analysis is an instantaneous approach, akin to the one traditionally used in mechanics, unlike the phase space study in quantifying recurrence through embedding time delay and dimension [12]. The observed bounded smaller variations in velocity, the bursty behavior arising from its sudden changes resulting in strong acceleration for the epileptic case may have physical significance in modelling finer aspects of seizure dynamics. As is well known, the transition from periodic motion to chaotic behavior can occur through several routes, like bi-stability in dynamical systems or due to the presence of short unstable periodic orbits separating these two motions. The unstable periodic orbits manifest in the Fourier power spectrum as a local enhancement of power at certain frequencies. For this purpose, we analyzed the Fourier power spectrum of the data sets, as shown in Fig 2. The analyzed time series is the accumulated fluctuations obtained from the cumulative sum of the series after subtracting the mean and normalizing the raw data. The Fourier power at higher frequencies showed power law behavior: $\mathrm{P}(\mathrm{f}) \sim \mathrm{f}^{-\alpha}$, as has been noted earlier. 
We report here for the first time the presence of short unstable periodic orbits (UPOs) at the boundary of alpha and beta brainwaves from the Fourier power spectra in EEG time series. In the power spectra, one observes the presence of small peaks in the mid frequency domain for the healthy subjects, not present in other samples, due to dynamical instability from the short periodic orbits $[29,30]$ or the so-called unstable orbits. It is worth emphasizing that these periodic orbits via the Gutzwiller formalism play an important role in determining the spectrum in the semi-classical approach [31]. Further, it has been reported that these short time periodic orbits determine the long-range spectral properties, and these are non-universal and system specific in nature [32]. Observed unstable periodic orbits of EEG recordings from the Fourier domain in Figs 2a, 2b reveal the presence of instability in normal brains and interestingly the instability appears at the boundary of low frequency alpha and beta wave bands of $8-14 \mathrm{~Hz}$ centered at $10 \mathrm{~Hz}$. The absence of the same in patients during seizure is discernible in the EEG signals, reported for the first time in this work. These deviations are observed from the power enhancement in the mid frequency domain in cases of healthy subjects and suppression of the same in the epilepsy case. The presence of short time periodic orbits highlights prominent features of the signals, as these are specific to certain functionalities in the brain [33]. Further, we analyzed the nature of unstable periodic orbits by reconstructing the signal from the Fourier transform of the UPO frequency region. The subsequent wavelet analysis, using the Morlet wavelet, having a Gaussian window with commensurate sinusoidal sampling functions, identified the wave front modulation as well as collapse and revival. From Figs $2 \mathrm{c}, 2 \mathrm{~d}$, the presence of wave packet collapse and revival is seen in the frequency range $8-14 \mathrm{~Hz}$ from our analysis, lying in the domain of alpha and beta brain waves. Reduced dominance of alpha and beta brain waves for epilepsy patients, as compared to healthy subjects and the oscillatory region dominance for these frequencies possibly indicates a transition from single particle to limit cycle behavior. Changes in this frequency range of the brain also reveals intra frequency transfer of energy becoming prominent during periods of seizure.

Coherence wave packet dynamics, characterized by collapse and revival, is observed starting from around $18 \mathrm{~Hz}$, clearly indicated from the Fourier domain in Figs 3a to 3e. The unambiguous wave packet dynamics is observed, with bursty power patterns visualized from the time frequency localization through wavelets, with higher power for patients during seizure as compared to healthy subjects. One also observes carrier wave modulation, visible for all subjects at $18 \mathrm{~Hz}$ with higher width for patients showing wave energy transfer in the intra frequency domain. It has been earlier reported that fast oscillations with frequencies $>40 \mathrm{~Hz}$ are a promising biomarker of the epileptogenic zone [24,26]. Oscillations in the gamma frequency band at $40 \mathrm{~Hz}$ of the EEG also play a critical role during behavioral wakefulness and cognition. Several experimental observations have been reported showing that neocortical oscillations at the gamma frequency (30-100 Hz) band, mainly around $40 \mathrm{~Hz}$, are involved in cognitive functions. Increase in gamma power typically appears during behaviors that are characterized by the cognitive processing of external precepts or internally generated thoughts and images. Gamma activity has also been observed during alert or attentive wakefulness, not only in humans, but also in animals. Well 
known coherence behavior is observed at the gamma wave region that connects the conscious to unconscious state of the brain. From our analysis in human EEG, it is observed from Figs. $3 \mathrm{f}$ to $3 \mathrm{i}$ that the coherence gets significantly enhanced around $40-45 \mathrm{~Hz}$.

Intra frequency energy transfer is observed, both at the junction of alpha and beta and beta and gamma waves, which is particularly prominent during the onset of seizure. The mechanism is established using the Heisenberg model, revealing that neutral turbulence is more prominent visa-vis viscous dissipation [34-36]. For quantification, we have analyzed the normalized global wavelet power with frequency in logarithmic scale and applied Heisenberg model fitting for both $\mathrm{K}=-5 / 3$ and $\mathrm{K}=-7$. The plots are shown in Fig 4 . The presence of neutral turbulence can be inferred through the Heisenberg model with $\mathrm{K}=-5 / 3$. It is prominent in comparison to the viscous dissipation regime $\mathrm{K}=-7$, as is observed from Fig. 4 of the supplementary material of this manuscript. The system is observed to make a transition at $10 \mathrm{~Hz}$, which is at the alpha- beta brain wave boundary. This $10 \mathrm{~Hz}$ transition regime in the neutral turbulence is the domain of observed unstable periodic orbits, as is evident from the Fourier space study. This is the frequency where the transfer of power occurs at the boundary of low frequency alpha and beta waves that becomes prominent for patients during seizure. We also analyzed the energy transfer phenomenon [37,38], by reconstructing the time series from the Fourier space, excluding the UPO range $8-14 \mathrm{~Hz}$ for use in the Heisenberg model. Once these characteristic frequencies are removed at $8-14 \mathrm{~Hz}$ in UPO range, the universal scale free behavior is manifest in the Heisenberg model. The observed transition at $10 \mathrm{~Hz}$ in the UPO range is identified as the energy transfer region, reported here for the first time. The fit of neutral turbulence is particularly good in the low frequency range up to $8 \mathrm{~Hz}$ for almost all subjects. It is evident that neutral turbulence is the mechanism of intra frequency energy transfer, both at the junction of alpha and beta, beta and gamma waves, that manifest during onset of seizure, when turbulence is observed to be significantly enhanced.

In conclusion, an instantaneous phase space approach to the study of EEG signals provides a unified picture of a diverse range of brain behavior. Manifestation of single particle oscillator like behavior at macroscopic level, progressively leading to limit cycle and bi-stability, as also wave packet collapse and revival, originating from the domain of unstable periodic orbits, opens up new avenues to explore and validate a number of features about brain dynamics surmised earlier. The fact that some of these features are much stronger in the epileptogenic zone and during the periods of seizure for patients shows the potential use of this instantaneous method for prediction and medical intervention. Analogy from the phase-space of oscillator dynamics reveals significantly enhanced potential energy contribution in the signals from the epileptogenic zone, as also in the patients before the occurrence of seizure. Coherence wave packet dynamics, evident from collapse and revival, is observed here for the first time, that emerges around $18 \mathrm{~Hz}$ inferred from the Fourier domain analysis of the phase space power-spectra. The Fourier analysis also reveals the unstable periodic orbits, observed around $10 \mathrm{~Hz}$ for healthy subjects, indicative of the presence of instability. The observed coherence gets significantly strengthened around $40-45 \mathrm{~Hz}$ as inferred from a local 
time frequency domain approach. Neutral turbulence is established as the mechanism of intra frequency energy transfer through the Heisenberg model.

\section{Materials}

For our analysis the EEG time series data of different subjects are obtained from the Department of Clinical Epileptology, University Hospital of Bonn, Germany [39]. The time series data consists of five sets, where first two datasets are extra-cranial recording of healthy volunteers during eyes open and eyes closed, whereas the intracranial recordings of patients during seizure free interval are obtained both from the hippocampal formation of the opposite hemisphere of the brain and recordings from within the epileptogenic zone. The fifth dataset is the recordings from patients obtained during the seizure activity. Each set contains 100 single channel EEG segments of 23.6 sec duration with each having 4097 recordings of electrical signal measured in $\mu \mathrm{V}$. These segments were selected and cut out from continuous multichannel EEG recordings after visual inspection for artifacts by a subject expert. The data acquisition system sampling rate is $173.61 \mathrm{~Hz}$. Since the selected patients belonged to different socioeconomic and age groups, we have carried out the mean subtraction for each single channel signal to remove the average part of the signal and then normalized it by dividing the standard deviation for further analysis.

\section{Appendix A. Supplementary material}

Supplementary material to this article can be found with this manuscript.

\section{Authors' contributions}

Mayukha Pal conceptualized the idea, performed the data analysis, and wrote the manuscript. Neeraj contributed in the development of the phase-space approach and content writing. Prasanta K. Panigrahi contributed in idea conceptualization, analysis direction, guidance, project supervision, review of the manuscript.

\section{Declaration of Competing Interest}

The authors declare that they have no known competing financial interest or personal relationships that could have appeared to influence the work reported in this paper.

\section{Acknowledgements}

This research work did not receive any specific grant from funding agencies in the public, commercial, or not-forprofit sectors however Neeraj is thankful to DST for the INSPIRE fellowship. The author MPal wishes to thank ABB Ability Innovation Center, Hyderabad for their support in this work and Dr. Abhinna Kumar Behera for his valuable discussion. The authors alone are responsible for the content and writing of the paper.

\section{Reference}

1. J. J. Wright and D. T. J. Liley, Dynamics of the brain at global and microscopic scales: Neural networks and the EEG. Behavioral and Brain Sciences 19, 285-320 (1996).

2. N. Pradhan, P. K. Sadasivan, S. Chatterji, D. Narayana Dutt, Patterns of Attractor dimensions of Sleep EEG. Comput. Biol. Med. 25 (5) 455-462 (1995).

3. J. Gao, J. Hu, W.-w Tung, Complexity measures of brain wave dynamics. Cognitive Neurodynamics 5, 171-182 (2011).

4. P. L. Nunez, Brain, mind, and the structure of reality. New York: Oxford University Press. (2010) 306, DOI:10.1093/acprof:oso/9780195340716.001.0001.

5. C. J. Stam, Nonlinear dynamical analysis of EEG and MEG: Review of an emerging field. Clinical Neurophysiology 116, 2266-2301 (2005).

6. F.H. Lopes Da Silva, W. Blanes, S.N. Kalitzin , J. Parra, P. Suffczynski, D.N. Velis, Epilepsies as dynamical diseases of brain systems: basic models of the transition between normal and epileptic activity. Epilepsia 44, 72-83 (2003).

7. E. Olbrich, P. Achermann, Analysis of oscillatory patterns in the human sleep EEG using a novel detection algorithm. J. Sleep Res. 14, 337-346 (2005).

8. P. Grassberger, I. Procaccia, Characterization of strange attractors. Phys Rev Lett 50, 346-349 (1983).

9. P. Grassberger, I. Procaccia, Estimation of the Kolmogorov entropy from a chaotic signal. Phys Rev A 28, 2591-2593 (1983). 
10. H. Chu, C. K. Chung, W. Jeong, K.-H. Cho, Predicting epileptic seizures from scalp EEG based on attractor state analysis. Computer Methods and Programs in Biomedicine 143, 75-87 (2017).

11. L.D. Iasemidis, H.P. Zaveri, J.C. Sackellares, W.J. Williams, Phase space analysis of EEG in temporal lobe epilepsy. Conf Proc IEEE Eng Med Biol Soc. Nov 4-7, New Orleans, LA, USA (1988).

12. A . Babloyantz, A . Destexhe, Low-dimensional chaos in an instance of epilepsy. Proc. Natl. Acad. Sci. USA 83 (10) 3513-3517 (1986).

13. M. B. MacIver, B. H. Bland, Chaos analysis of EEG during isoflurane-induced loss of righting in rats. Frontiers in Systems Neuroscience 8 (203) 1-8 (2014). Doi: 10.3389/fnsys.2014.00203

14. H. Korn, P. Faure, Is there chaos in the brain? II. Experimental evidence and related models. C. R. Biologies 326, 787840 (2003).

15. N. Kannathal, J. Chee, E.R. Kenneth, K. Lim, O. H. Tat, Chaotic Analysis of Epileptic EEG Signals. DOI: 10.1007/9783-319-02913-9_166.

16. J. Martinerie, C. Adam, M. L. V. Quyen, M. Baulac, S. Clemenceau, B. Renault, F. Varela, Epileptic seizures can be anticipated by non-linear analysis. Nat Med 4, 1173-1176 (1998).

17. V. K. Jirsa, W. C. Stacey, P. P. Quilichini, A. I. Ivanov, C. Bernard, On the nature of seizure dynamics. Brain 137, 22102230 (2014).

18. M. Pal, P. M. Rao, EEG Epilepsy Seizure Signal Characterization through theories of Random Matrix Analysis. $8^{\text {th }}$ International Conference on Latest Trends in Engineering and Technology (ICLTET'2016) May 5-6 2016 Dubai (UAE), ISBN : 978-93-84422-65-3 (2016).

19. P. J. Franaszczuk, K. J. Blinowska, Linear Model of Brain Electrical Activity - EEG as a Superposition of Damped Oscillatory Modes. Biol. Cybern. 53, 19-25 (1985).

20. K. B. Doelling, M. F. Assaneo, D. Bevilacqua, B. Pesaran, D. Poeppel, An oscillator model better predicts cortical entrainment to music. Proc. Natl. Acad. Sci. USA 116 (20) 10113-10121 (2019).

21. B. J. Griffiths, G. Parisha, F. Roux et. al., Directional coupling of slow and fast hippocampal gamma with neocortical alpha/beta oscillations in human episodic memory. Proc. Natl. Acad. Sci. USA 116 (43) 21834-21842 (2019).

22. U. V. Borodina, R. R. Aliev, Wavelet spectra of visual evoked potentials: Time course of delta, theta, alpha and beta bands. Neurocomputing 1219, 551-555 (2013).

23. G. A. Worrell, L. Parish, S. D. Cranstoun, R. Jonas, G. Baltuch, B. Litt, High-frequency oscillations and seizure generation in neocortical epilepsy. Brain 127, 1496-1506 (2004).

24. E. Urrestarazu, R. Chander, F. Dubeau, J. Gotman, Interictal high-frequency oscillations $(100-500 \mathrm{~Hz})$ in the intracerebral EEG of epileptic patients. Brain 130, 2354-2366 (2007).

25. G. T. Finnerty, J.G.R. Jefferys, 9-16 Hz Oscillation Precedes Secondary Generalization of Seizures in the Rat Tetanus Toxin Model of Epilepsy. Journal of Neurophysiology 83 (4) 2217-2226 (2000).

26. F. Deeba, P. Sanz-Leon, P. A. Robinson, Effects of physiological parameter evolution on the dynamics of tonic-clonic seizures. PLoS ONE 15 (4) e0230510 (2020). Doi: 10.1371/journal.pone.0230510

27. E. Pavlovskaia, J. Ing, M. Wiercigroch, S. Banerjee, Complex dynamics of bilinear oscillator close to grazing. Int. J. Bifurcation Chaos 20 (11) 3801-3817 (2010).

28. R. J. Macgregor, Quantum mechanics and brain uncertainty. Journal of Integrative Neuroscience 5 (3) $373-380$ (2006).

29. J. H. Hannay, A. M. O. de Almeida, Periodic orbits and a correlation function for the semiclassical density of states. J. Phys. A 17, 3429 (1984).

30. E. Heller, Bound-State Eigenfunctions of Classically Chaotic Hamiltonian Systems: Scars of Periodic Orbits. Phys. Rev. Lett. 53, 1515 (1984).

31. M. S. Santhanam, J. N. Bandyopadhyay, Spectral Fluctuations and 1/f Noise in the Order-Chaos Transition Regime. Phys. Rev. Lett. 95, 114101 (2005).

32. F. Haake, Quantum Signatures of Chaos. (Springer-Verlag, Berlin, 2000), 2nd ed.

33. M. V. Berry, Uniform asymptotic smoothing of Stokes's discontinuities. Proc. R. Soc. A 422,7 (1989).

34. A. N. Kolmogorov, The local structure of turbulence in incompressible viscous fluid for very large Reynolds number. Pro. R. Soc. London, Ser. A 434, 15-17 (1991).

35. W. Heisenberg, On the theory of statistical and isotropic turbulence. Proc. R. Soc. London, Ser. A 195(1042), $402-406$ (1948).

36. M. Farge, N. Kevlahan, V. Perrier, and E. Goirand, Wavelets and turbulence. Proc. IEEE 84(4), 639-669 (1996).

37. U. Das, H. S. S. Sinha, S. Sharma, H. Chandra, S. K. Das, Fine structure of the low-latitude mesospheric turbulence. J. Geophys. Res. 114, D10111 (2009). 
38. A. K. Behera, A. S. Iyengar, P. K. Panigrahi, Non-stationary dynamics in the bouncing ball: A wavelet perspective. Chaos 24 (4) 043107 (2014).

39. R. G. Andrzejak, K. Lehnertz, F. Mormann, C. Rieke, P. David, C. E. Elger, Indications of nonlinear deterministic and finite-dimensional structures in time series of brain electrical activity: Dependence on recording region and brain state. Physical Review E 64, 061907 (2001).

\section{Figures}

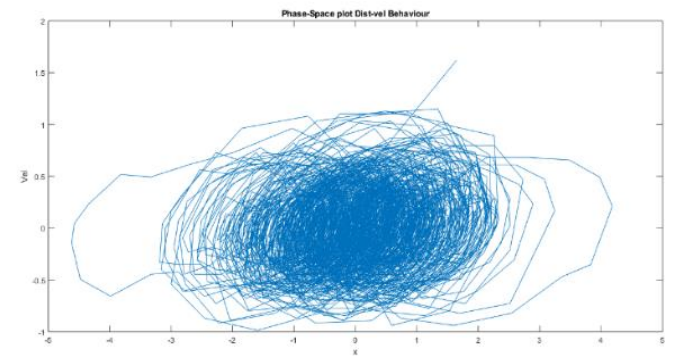

(a) Phase space plot of healthy (eye open) individual's EEG signals, with the potential as coordinate and its instantaneous change as velocity, clearly depicting periodic motion like that of a linear oscillator, with multiple loops indicating multiperiodic nature.

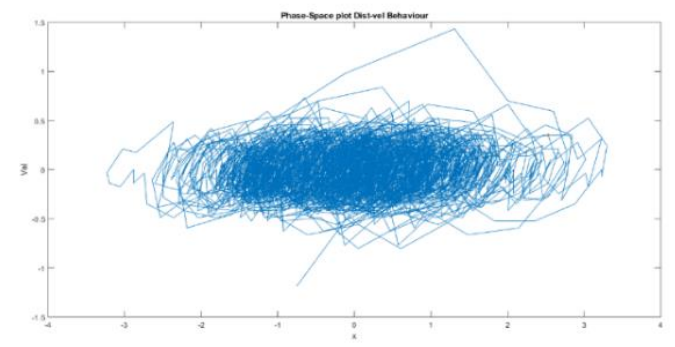

(c) Phase space plot of patient's signal (without seizure) and signal from the epileptogenic zone show much stronger ellipticity, reduced kinetic energy and velocity spread.

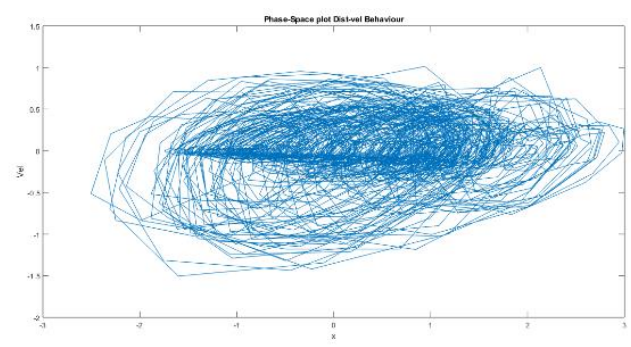

(e) From the phase space analysis of other representative channel $\left(40^{\text {th }}\right)$ for patients during the seizure, bimodal structures are observed for a few orbits which are spread out,

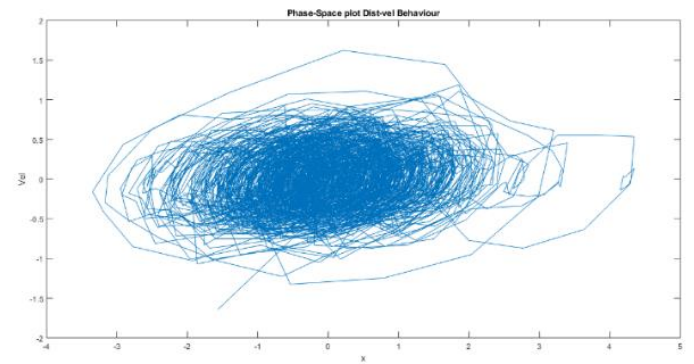

(b) Phase space plot for the eye closed healthy subjects shows multi-periodic motion with higher ellipticity, indicative of reduced kinetic energy.

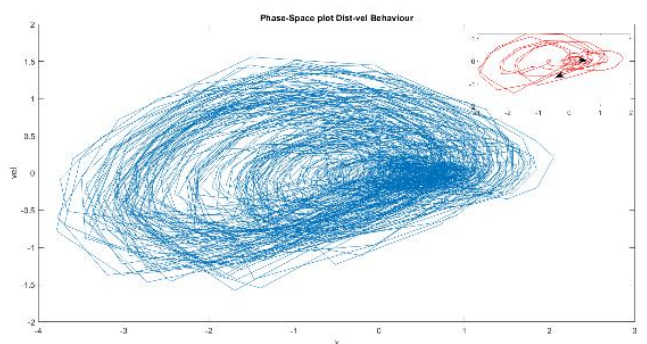

(d) Phase space plot of patient's signal during the period of seizure shows behavior like that of a coupled oscillator with emergence of limit point. The inset figure shows the same with only 200 data points, the arrow showing its evolution.

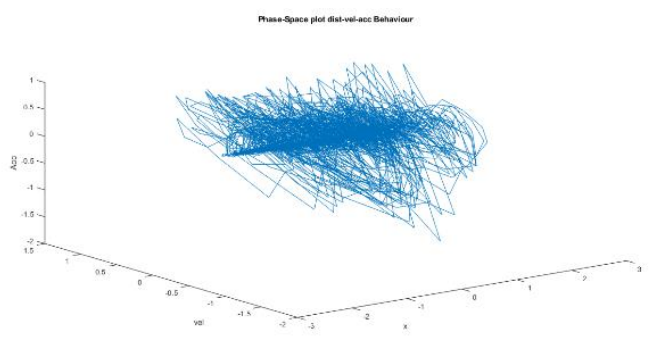

(f) For patients during seizure from the $40^{\text {th }}$ channel recordings of coordinate, velocity and acceleration in phase space 3D reveal much less spread in velocity, rapid irregular 
while most of them are tightly packed, showing significant increase in potential energy. variations in a much bigger fraction of closed orbits and much more potential energy as compared to Fig 1c.

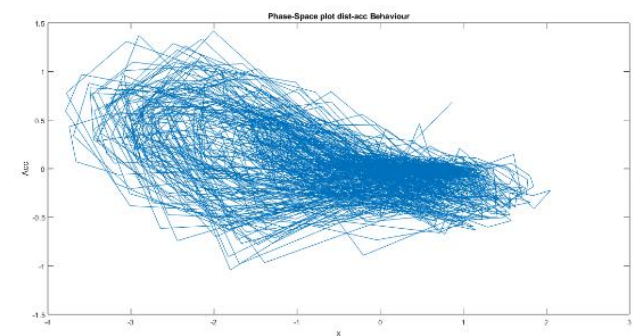

(g) For patients during seizure from the $1^{\text {st }}$ channel recordings between the coordinate and acceleration depicting the increase in the potential energy and corresponding decreased kinetic energy, possibly indicative of a limit cycle behavior during the period of seizure.

Figure-1: Phase Space diagram for different subjects.

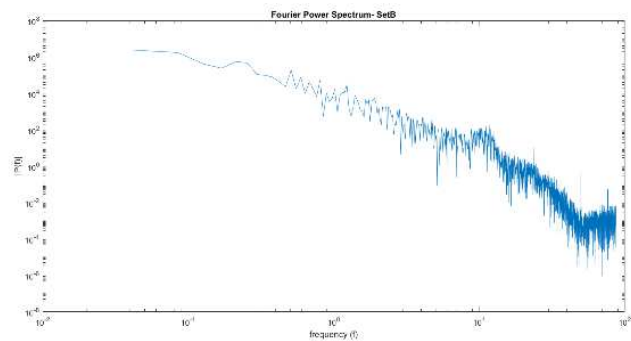

(a) Healthy (eye closed) subject's Fourier power spectra depicting UPO at the boundary of alpha and beta brain waves $8-14 \mathrm{~Hz}$ centered at $10 \mathrm{~Hz}$.

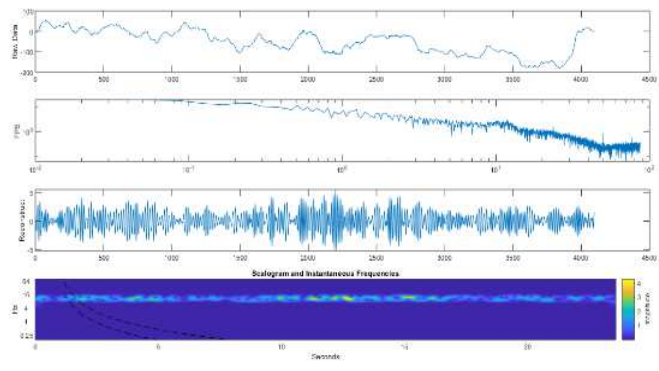

(c) Reconstructed signal of the UPO range with time frequency localization scalogram for a healthy subject during eye closed showing strong oscillatory regions.

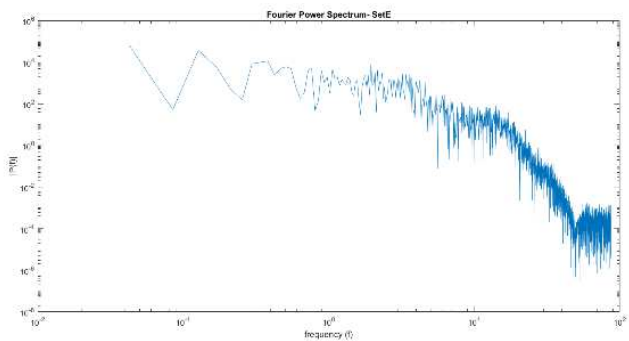

(b) From patient's (during seizure) Fourier power spectra the reduction in UPO is discernible and a characteristic feature for subject state classification.

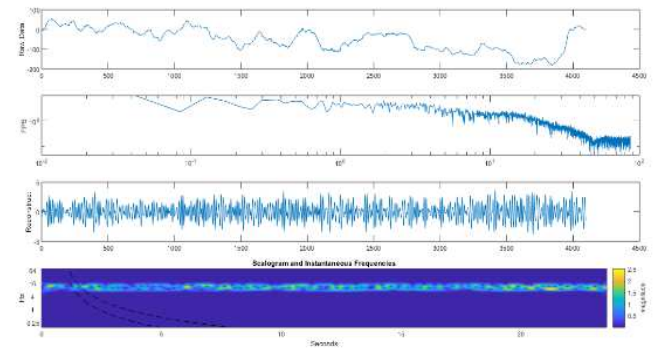

(d) Reconstructed signal of the UPO range with time frequency localization for a patient during seizure shows reduced oscillatory regions.

Figure-2: Fourier power spectra of all datasets, showing local power enhancement for healthy subjects in the mid-frequency domain, not present in the patient's dataset, revealing a much broader frequency distribution with significant power. Also, from Figs $2 \mathrm{c}$ and $2 \mathrm{~d}$ the reconstructed signal for the UPO region from Fourier power spectra shows oscillatory regions and bursty behavior. 

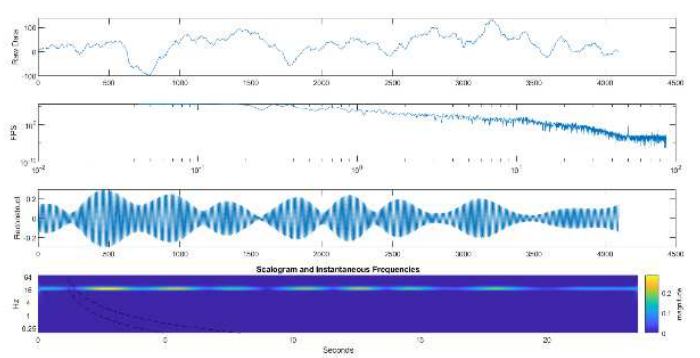

(a) Reconstructed signal at $18 \mathrm{~Hz}$ depicting strong wave packet dynamics of collapse and revival for healthy subjects (eye open), as also carrier wave modulations.

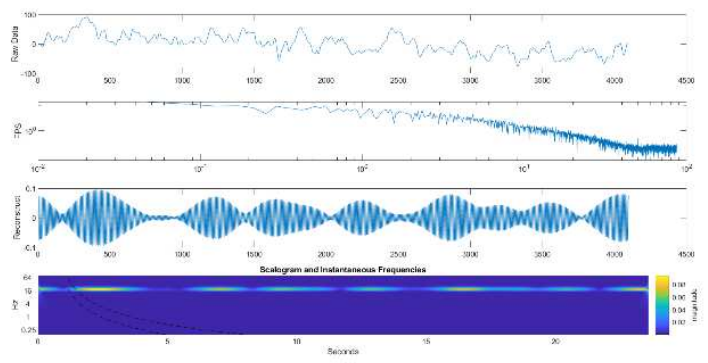

(c) Reconstructed signal at $18 \mathrm{~Hz}$ showing wave packet dynamics with higher power observed from time frequency localization for patients (no seizure) signal from hippocampal zone.

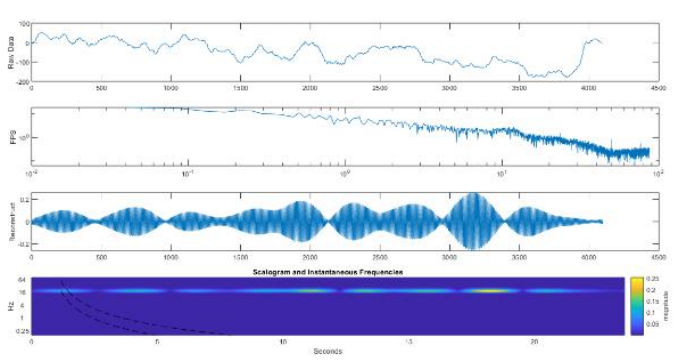

(b) Reconstructed signal at $18 \mathrm{~Hz}$ for healthy subject (eye closed) showing wave packet dynamics with reduced carrier wave modulation.

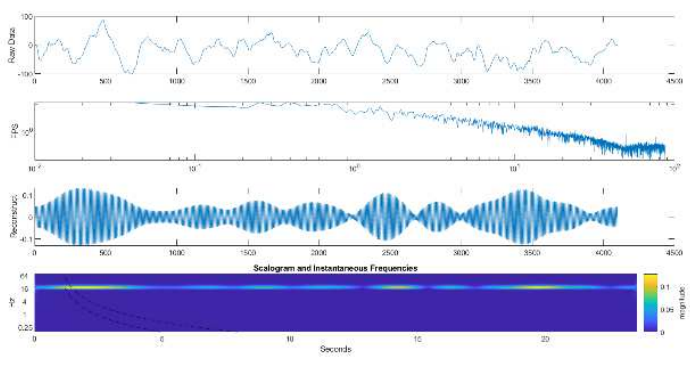

(d) Reconstructed signal at $18 \mathrm{~Hz}$ showing wave packet dynamics, wider carrier wave with higher power observed from time frequency localization for patients (no seizure) signal from epileptogenic zone.
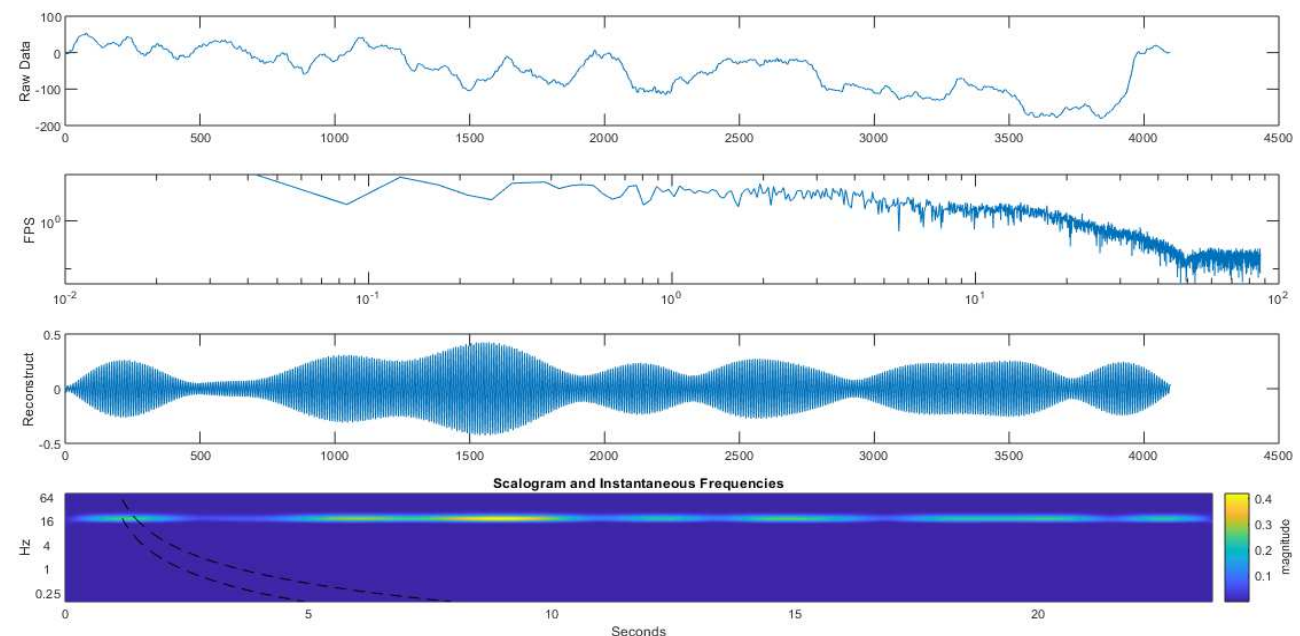

(e) Reconstructed signal at $18 \mathrm{~Hz}$ depicts wave packet dynamics with much higher power observed from time frequency localization for patients during the period of seizure. The carrier wave modulation gets wider for patients suffering from seizure in comparison to healthy subjects 


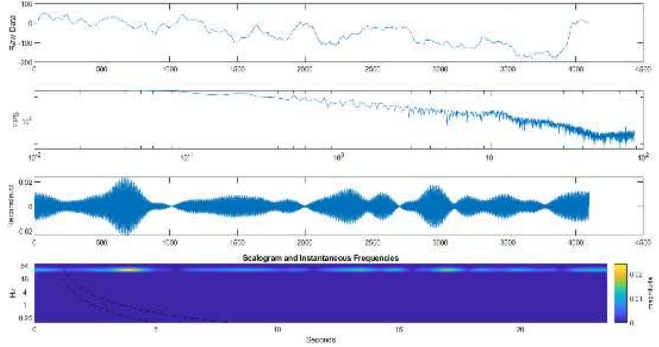

(f) Reconstructed signal at $40 \mathrm{~Hz}$ depicting stronger interference and wave packet dynamics, compared to that of $18 \mathrm{~Hz}$ for healthy subjects (eye closed).

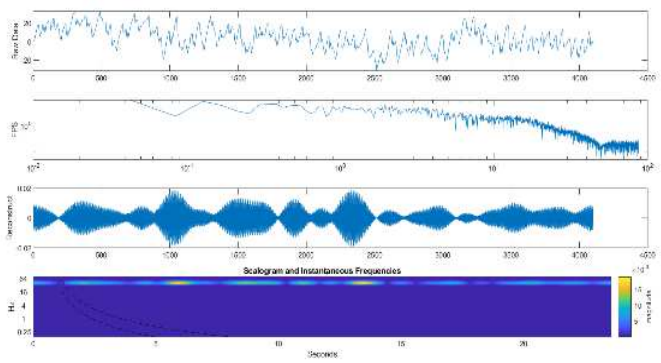

(h) Reconstructed signal at $40 \mathrm{~Hz}$ showing stronger wave packet dynamics with higher power observed from time frequency localization for patients during seizure compared to healthy subjects.

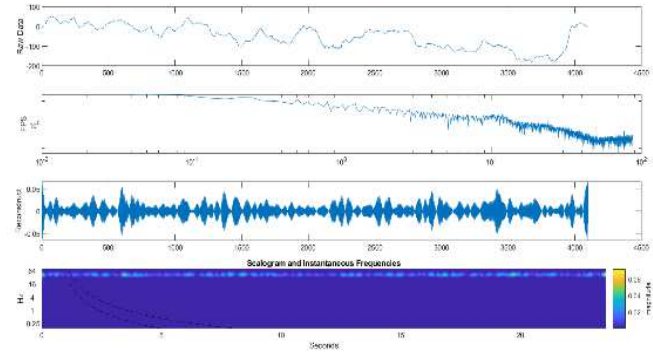

(g) Reconstructed signal at $40-45 \mathrm{~Hz}$ showing stronger interference as compared to $18 \mathrm{~Hz}$ for healthy subjects (eye closed) with manifestation of wave superposition.

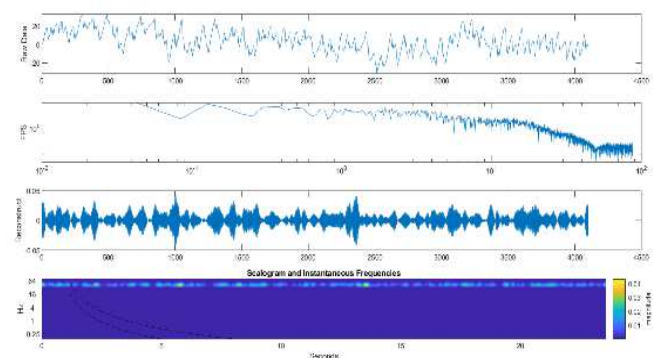

(i) Reconstructed signal at $40-45 \mathrm{~Hz}$ showing stronger interference and wave packet dynamics with higher power, faster superposition of waves observed from time frequency localization for patients during seizure.

Figure-3: Reconstructed signal at $18 \mathrm{~Hz}$ showing wave packet dynamics with carrier wave modulation showing energy transfer. Figs. from $3 \mathrm{f}$ to $3 \mathrm{i}$ show reconstructed signals at $40 \mathrm{~Hz}$ and $40-45 \mathrm{~Hz}$ showing stronger wave packet dynamics.

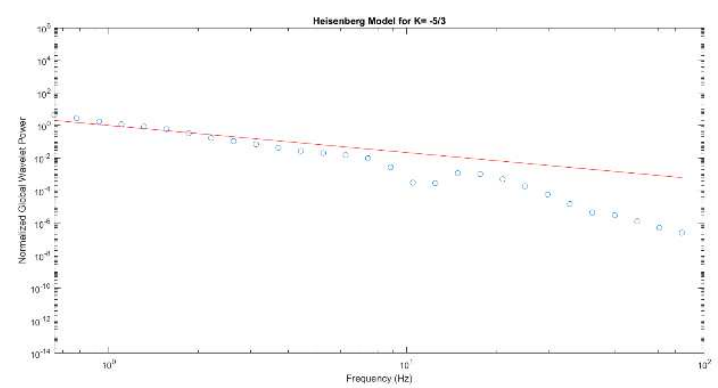

(a) Heisenberg model's energy transfer phenomena of neutral turbulence $(K=-5 / 3)$ from the reconstruction signal without the UPO range $8-14 \mathrm{~Hz}$ frequency for healthy (eye closed).

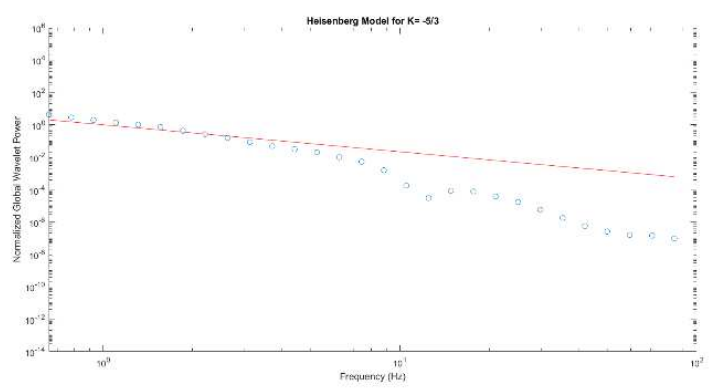

(b) Heisenberg model's energy transfer phenomena of neutral turbulence $(K=-5 / 3)$ from the reconstruction signal without the UPO range $8-14 \mathrm{~Hz}$ frequency for patients with no seizure (epileptogenic zone of brain). 


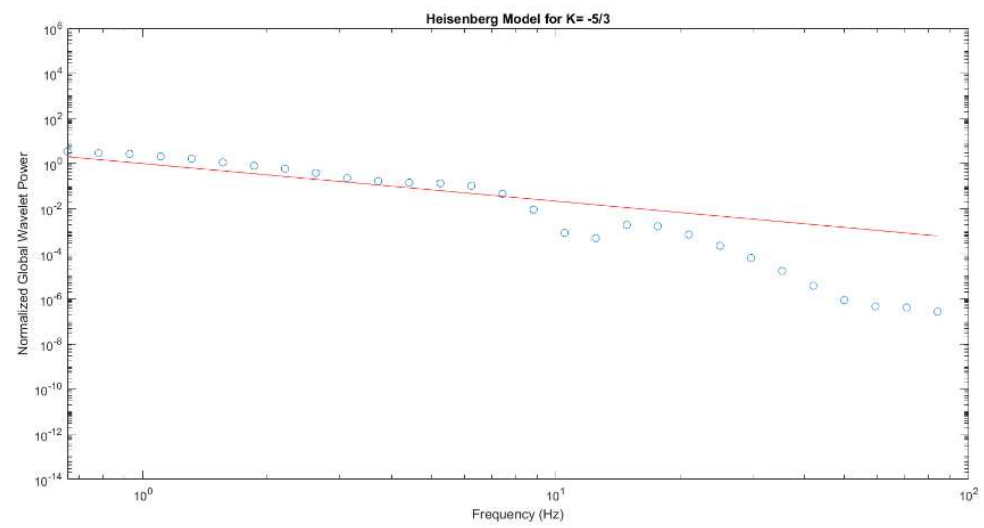

(c) Fit of the Heisenberg model's energy transfer phenomena of neutral turbulence $(\mathrm{K}=-5 / 3)$ from the reconstructed signal without the UPO range $8-14 \mathrm{~Hz}$ of frequency for patients during the period of seizure. Energy transfer phenomena belong to neutral turbulence at the junction of alpha and beta wave and beta and gamma wave, indicated from the excellent fit at lower frequencies.

Figure-4: Heisenberg model for different subjects without UPO component. 


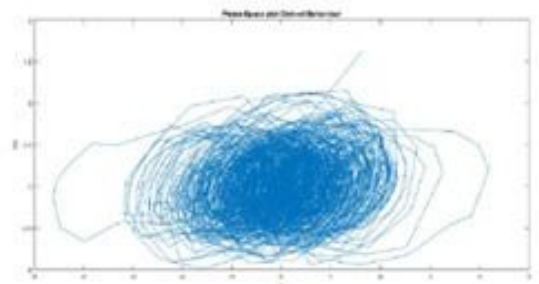

(a) Phase space plot of healthy (eye open) individual's EEG signals, with the potential as coordinate and its instantaneous change as velocity, clearly depicting periodic motion like that of a linear oscillator, with multiple loops indicating multiperiodic nature

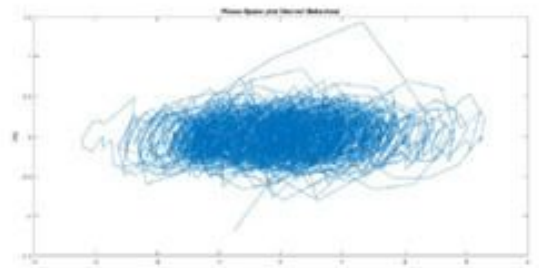

(c) Phase space plot of patient's signal (without seizure) anp signal from the epileptogenic zone show much stronger ellipticity, reduced kinetic energy and velocity spread

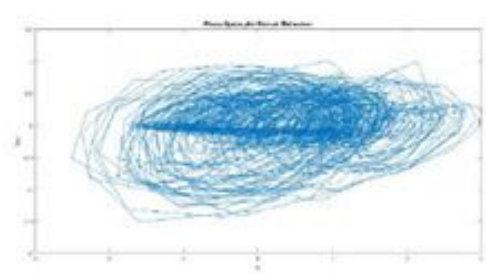

(e) From the phase space analysis of other representative channel $\left(40^{\text {th }}\right)$ for patients during the seizure, bimodal structures are observed for a few orbits which are spread out, while most of them are tightly packed, showing significant increase in potential energy.

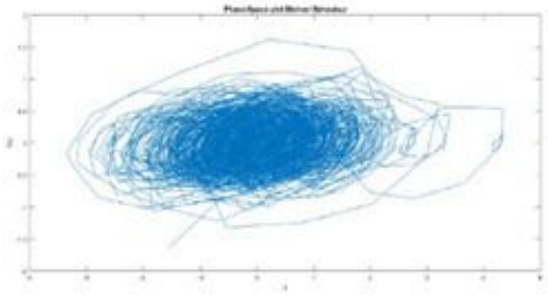

(b) Phase space plot for the eye closed healthy subjects shows multi-periodic motion with higher ellipticity, indicative of reduced kinetic energy.

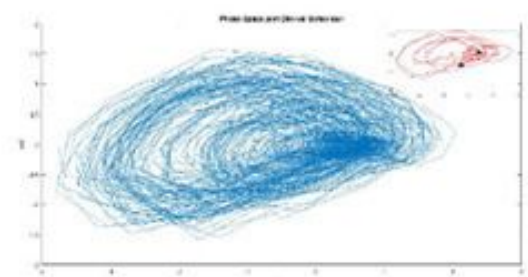

(d) Phase space plot of patient's signal during the period of seizure shows behavior like that of a coupled oscillator with emergence of limit point. The inset figure shows the same with only 200 data points, the arrow showing its evolution.

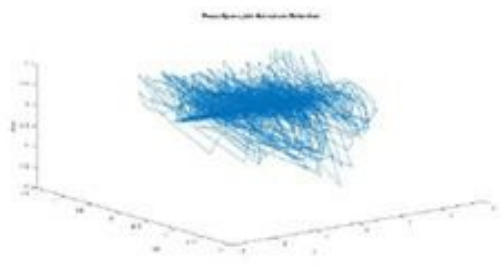

(f) For patients during seizure from the $40^{\star}$ channel recordings of coordinate, velocity and acceleration in phase space $3 \mathrm{D}$ reveal much less spread in velocity, rapid irregular variations in a much bigger fraction of closed orbits and much more potential energy as compared to Fig 1c.

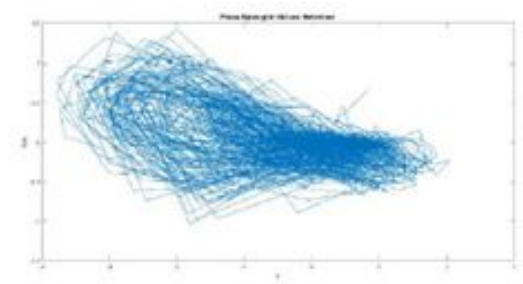

(g) For patients during seizure from the $1^{\text {th }}$ channel recordings between the coordinate and acceleration depicting the increase in the potential energy and corresponding decreased kinetic energy, possibly indicative of a limit cycle behavior during the period of seizure.

\section{Figure 1}

\section{Phase Space diagram for different subjects}




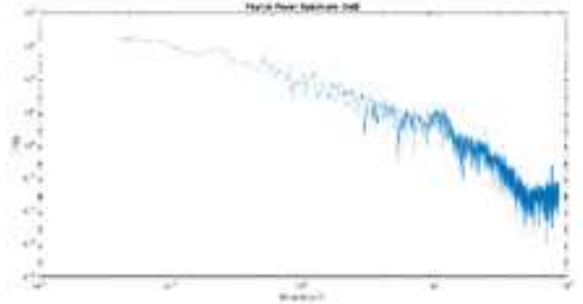

(a) Healthy (eye closed) subject's Founier power spectra depicting UPO at the botindary of alpha and beta brain waves $8-14 \mathrm{~Hz}$ centered at $10 \mathrm{~Hz}$

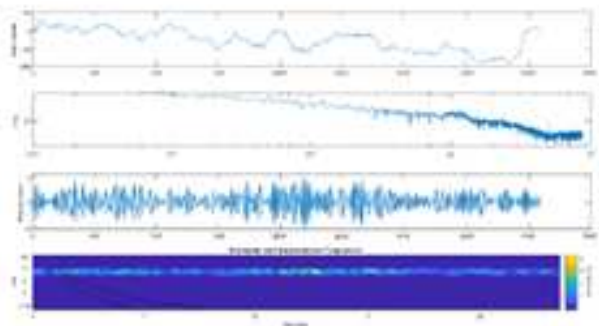

(c) Reconstructed signal of the UPO range with time frequency localization scalogram for a bealthy subject during eye closed showing strong oscillatory regions.

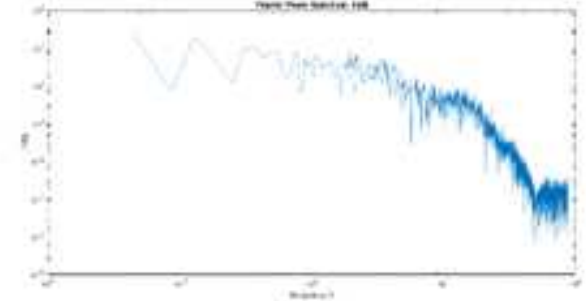

(b) From patient's (during seizure) Founier power spectra the reduction in UPO is discernible and a characteristic feature for subject state classification.

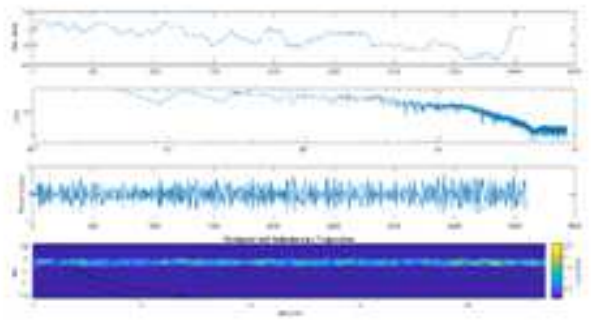

(d) Reconstructed signal of the UPO range with time frequency localization for a patient during seizure shows reduced oscillatory regions.

\section{Figure 2}

Fourier power spectra of all datasets, showing local power enhancement for healthy subjects in the midfrequency domain, not present in the patient's dataset, revealing a much broader frequency distribution with significant power. Also, from Figs $2 \mathrm{c}$ and $2 \mathrm{~d}$ the reconstructed signal for the UPO region from Fourier power spectra shows oscillatory regions and bursty behavior. 


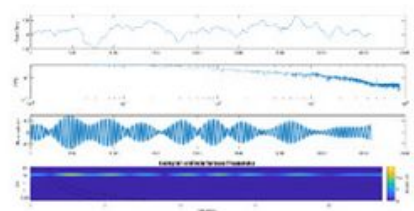

(a) Reconstructed signal at $18 \mathrm{~Hz}$ depicting strong wave packet dynamics of collapse and revival for healthy subjects (eye open), as also carrier wave modulations.

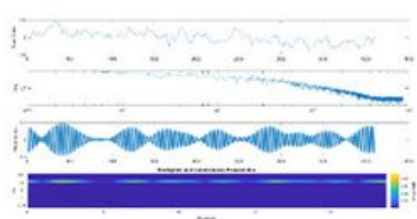

(c) Reconstructed signal at $18 \mathrm{~Hz}$ showing wave packet dynamics with higher power observed from time frequency localization for patients (no seizure) signal from hippocamp zone.

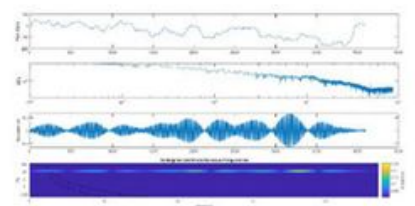

(b) Reconstructed signal at $18 \mathrm{~Hz}$ for healthy subject (eye losed) showing wave packet dynamics with reduced camier wave modulation.

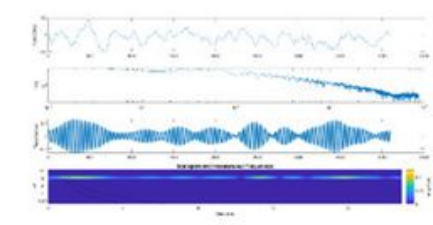

(d) Reconstructed signal at $18 \mathrm{~Hz}$ showing wave packet dynamics, wider carrier wave with higher power observed from time frequency localization for patients (no seizure) signal from epileptogenic zone.
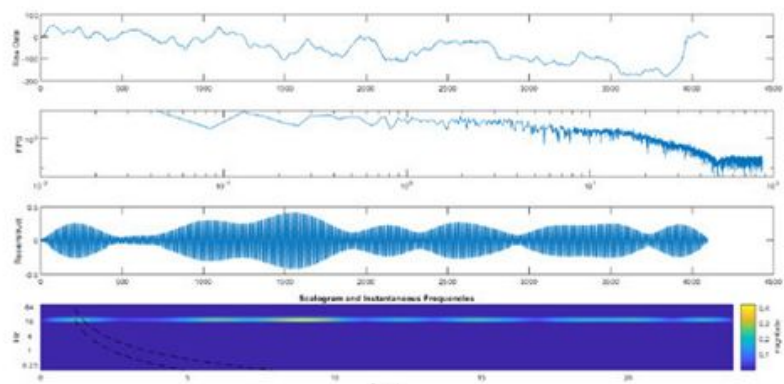

(e) Reconstructed signal at $18 \mathrm{~Hz}$ depicts wave packet dynamics with much higher power observed from time frequency localization for patients during the period of seizure. The carrier wave modulation gets wider for patients suffering from seizure in comparison to healthy subjects

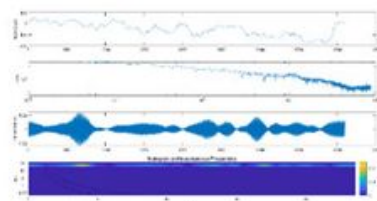

(f) Reconstructed signal at $40 \mathrm{~Hz}$ depicting stronger interference and wave packet dynamics, compared to that of $18 \mathrm{~Hz}$ for healthy subjects (eye closed).

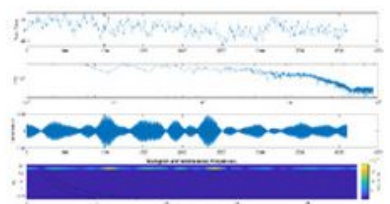

(h) Reconstructed signal at $40 \mathrm{~Hz}$ showing stronger wave packet dynamics with higher power observed from time frequency localization for patients during seizure compared to healthy subjects.

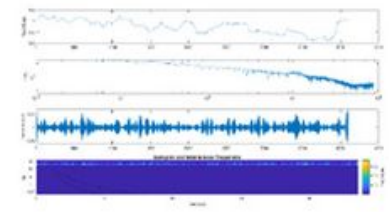

g) Reconstructed signal at $40.45 \mathrm{~Hz}$ showing stronger interference as compared to $18 \mathrm{~Hz}$ for healthy subjects (eye closed) with manifestation of wave superposition.

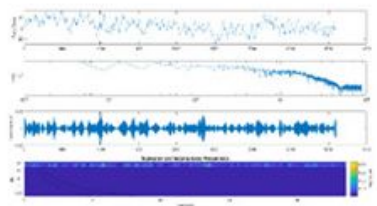

(1) Reconstructed signal at $40-45 \mathrm{~Hz}$ showing stronger interference and wave packet dynamics with higher power. faster superposition of waves observed from time frequency localization for patients during seizure.

\section{Figure 3}

Reconstructed signal at $18 \mathrm{~Hz}$ showing wave packet dynamics with carrier wave modulation showing energy transfer. Figs. from $3 \mathrm{f}$ to $3 \mathrm{i}$ show reconstructed signals at $40 \mathrm{~Hz}$ and $40-45 \mathrm{~Hz}$ showing stronger wave packet dynamics. 


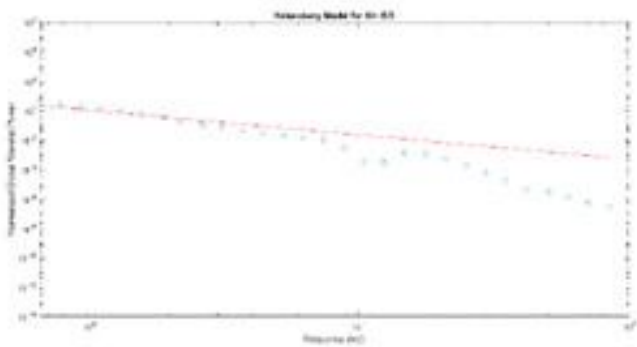

(a) Heisenberg model's energy transfer phenomena of neutral turbulence $(K=5 / 3)$ from the reconstruction signal without the UPO range $8-14 \mathrm{~Hz}$ frequency for healthy (eye closed).

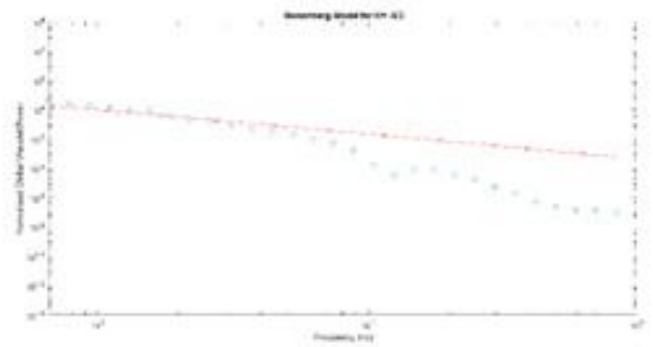

(b) Heisenberg model's energy transfer phenomena of neutral turbulence $(\mathrm{K}=5 / 3)$ from the reconstruction signal without the UPO range $8-14 \mathrm{~Hz}$ frequency for patients with no seizure (epileptogenic zone of brain).

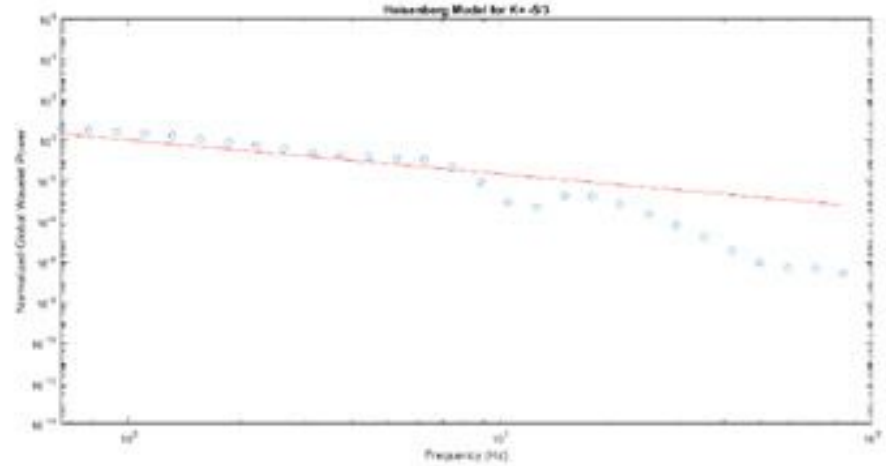

(c) Fit of the Heisenberg model's energy transfer phenomena of neutral turbulence ( $\mathrm{K}=-5 / 3)$ from the reconstructed signal without the UPO range $8-14 \mathrm{~Hz}$ of frequency for patients during the period of seizure. Energy transfer phenomena belong to neutral turbulence at the junction of alpha and beta wave and beta and gamma wave, indicated from the excellent fit at lower frequencies.

\section{Figure 4}

Heisenberg model for different subjects without UPO component.

\section{Supplementary Files}

This is a list of supplementary files associated with this preprint. Click to download.

- SuppIMatOscillatorNature.pdf 